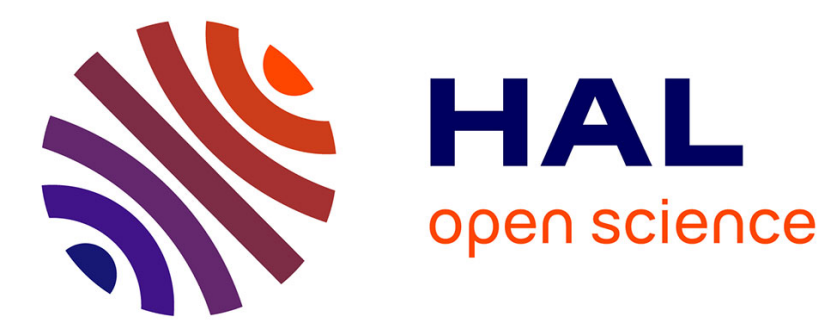

\title{
Émotions et dynamique des représentations sociales
}

Boumédine Bouriche

\section{To cite this version:}

Boumédine Bouriche. Émotions et dynamique des représentations sociales. 55ème Congrès de la Société Française de Psychologie, Sep 2013, Lyon, France. hal-01693558

\section{HAL Id: hal-01693558 https://hal-amu.archives-ouvertes.fr/hal-01693558}

Submitted on 18 Mar 2019

HAL is a multi-disciplinary open access archive for the deposit and dissemination of scientific research documents, whether they are published or not. The documents may come from teaching and research institutions in France or abroad, or from public or private research centers.
L'archive ouverte pluridisciplinaire HAL, est destinée au dépôt et à la diffusion de documents scientifiques de niveau recherche, publiés ou non, émanant des établissements d'enseignement et de recherche français ou étrangers, des laboratoires publics ou privés. 


\section{EMOTIONS ET DYNAMIQUE DES REPRESENTATIONS SOCIALES}

Boumédienne BOURICHE - (boumedienne.bouriche@univ-amu.fr)

Aix Marseille Université 


\section{THEORIE DES REPRESENTATIONS SOCIALES (RS)}

2 approches principales (Abric, 1976; Doise, 1986) :

Forme de connaissance sociale, pensée du sens commun, socialement élaborée et partagée.

" «Vision fonctionnelle du monde», cadre de référence pour comprendre la réalité sociale et donner du sens aux conduites.

- Cadre de référence commun modulé par des variations individuelles. 


\section{THEORIE DES \\ REPRESENTATIONS SOCIALES \\ (RS)}

La Théorie du Noyau Central TNC (Abric, 1976) :

Système central : Structuration (signification symbolique), Stabilité (préservation).

Système périphérique : Concrétisation, Régulation, Défense. 


\section{LES EMOTIONS}

Etat affectif : automatique, valence, intensité, brièveté.

Affects : formes incipientes (Rimé, 2005).

Approche cognitive de l'émotion (Arnold, 1960; Frijda 1986; Lazarus, 1991; Schachter, 1964 ; Scherer 1984) .

Évaluation globale d'un objet de la réalité sociale. 


\section{REPRESENTATIONS SOCIALES ET EMOTIONS}

Perspective structuraliste : composante affective des RS (Banchs, 1996 ; Roque, 1998 ; Methivier, 2012).

Point d'origine : Nexus (Rouquette, 1994; Campos \& Rouquette, 2000; Kalampalikis, 2001; Delouvée, 2006) .

- Compréhension de l'organisation interne (« logique affective » Deschamps et Guimelli, 2002; « sentiments » Lheureux \& Guimelli, 2009).

«La composante émotionnelle des $R S$ constitue un aspect important à prendre en compte si l'on veut mieux comprendre leur organisation et leur fonctionnement interne.» (Guimelli \& Rimé, 2009). 


\section{REPRESENTATIONS SOCIALES ET EMOTIONS}

Perspective structuraliste : obstacle au rapprochement théorique.

Double opposition : caractère collectif/individuel et stabilité/brièveté.

« Quand on les communique, émotions et cognitions se trouvent dans une continuité intériorisée ; elles sont les facettes d'une même expérience psychique, le résultat d'un même processus. " (Moscovici 2005).

Ce dernier point nous invite à privilégier une approche fonctionnaliste des relations entre émotions et RS. 


\section{REPRESENTATIONS SOCIALES ET EMOTIONS}

Analyse fonctionnelle :

EMOTIONS

\begin{tabular}{|c|c|c|}
\hline SAVOIR & VISION COMMUNE & $\begin{array}{l}\text { EXPERIENCE } \\
\text { INDIVIDUELLE }\end{array}$ \\
\hline ACTION & $\begin{array}{l}\text { ORIENTATION DES } \\
\text { CONDUITES }\end{array}$ & $\begin{array}{l}\text { AJUSTEMENT DES } \\
\text { CONDUITES }\end{array}$ \\
\hline IDENTITE & $\begin{array}{l}\text { APPARTENANCE DE } \\
\text { GROUPE }\end{array}$ & IMAGE DE SOI \\
\hline COMMUNICATION & $\begin{array}{c}\text { FORMATION ET } \\
\text { TRANSFORMATION }\end{array}$ & PARTAGE SOCIAL \\
\hline DYNAMIQUE & \multicolumn{2}{|c|}{$\begin{array}{l}\text { MEME ORIGINE D'ACTIVATION : } \\
\text { ECART PERCU / REALITE }\end{array}$} \\
\hline
\end{tabular}




\section{REPRESENTATIONS SOCIALES ET EMOTIONS}

Forte homologie fonctionnelle insuffisamment soulignée.

Mêmes enjeux adaptatifs, à la fois épistémiques et identitaires.

Processus de production de sens à même finalité : élaboration « d'un monde habituel, familier, rassurant que les individus partagent » et préservation des « digues symboliques » héritées du passé (Rimé, 2005).

Perspective fonctionnaliste : participation à une même dynamique de continuité dans le rapport à la réalité sociale (Moscovici, 1969; Rimé, 2005). 


\section{REPRESENTATIONS SOCIALES ET EMOTIONS}

Rapprochement théorique entre RS et Emotions :

RS = « système de pré-décodage de la réalité, qui détermine un système d'anticipations et d'attentes » et processus d'ancrage (assignation de sens) (Abric, 1994).

Activation émotionnelle et Sensemaking (Weick, 1995) = signal d'une défaillance du système d'attentes. «Opérations perceptivo-cognitives complexes de surveillance et d'évaluation de la réalité» (Rimé, 2005).

Activation émotionnelle $=$ annonce de déclenchement potentiel $\mathrm{du}$ processus d'ancrage. 


\section{REPRESENTATIONS SOCIALES ET EMOTIONS}

À partir des propositions de Weick (1995) et des propriétés de la TNC (Abric, 1976; Flament, 1994) :

Émotions = dispositions évaluatives individuelles basées sur l'application des cadres de référence communs que constituent les RS.

Signaux d'évaluation de la qualité des systèmes de représentation dans leur fonction d'anticipation de la réalité.

Proposition conciliable avec une approche des RS tant du point de vue de leur genèse (Moscovici, 1976) que de leur dynamique (Flament, 1994). 


\section{REPRESENTATIONS SOCIALES ET EMOTIONS}

Défaillance de sens liée à la défaillance du système d'attentes et processus d'attribution :

- Contexte productif d'une attribution : incohérences et variations de l'environnement (Heider, 1958), évènements inattendus (Bohner et al., 1988 ; Hastie, 1984 ; Langer et al, 1978).

RS $=$ « toile de fond des processus d'attribution » (Deschamps \& Clémence, 1987).

- Possible de distinguer les effets des émotions sur l'activation et la dynamique d'un objet de représentation. L'attribution des émotions aux éléments du champ représentationnel permet de rendre compte de leur niveau d'activation. 


\section{REPRESENTATIONS SOCIALES ET EMOTIONS}

Hypothèse de production affective :

- H1 attentes (V.I.1) et valence : valence directement proportionnelle au niveau de conformité aux attentes.

Hypothèses d'activation des croyances selon la valence (V.I.2) :

H2 effet global de justification : effet d'activation plus marqué des croyances centrales.

H3 : effet de valence positive : effet d'activation plus marqué sur les croyances centrales.

H4 : effet de valence négative : effet d'activation plus marqué sur les croyances périphériques.

$\sum$ Introduction $>$ Hypothèses 


\section{REPRESENTATIONS SOCIALES ET EMOTIONS}

Hypothèses de dynamique représentationnelle selon la valence (V.I.2) :

H5 : résistance des croyances centrales : cadre de référence commun.

H6 : effet de valence positive : modulations orientées sur les croyances centrales (fonction justificatrice).

H7 : effet de valence négative : modulations orientées sur les croyances périphériques (fonction régulation et de défense).

$\sum$ Introduction $>$ Hypothèses 


\section{REPRESENTATIONS SOCIALES ET EMOTIONS}

RS du travail en équipe.

Participants : 43 étudiants GEA (M : 20.76, SD : 1.30).

Situation de travail en équipe : simulation de gestion sur 3 jours.

Evènements imprévus ou provoqués.

$\sum$ Introduction $\rangle$ Hypothèses $\rangle$ Méthodologie


Matériel et procédure.

\section{PHASE 2}

Analyse de la structure de la RS du travail en équipe :

- Questionnaire de caractérisation +

- Test de centralité (Test d'Indépendance au Contexte)

\section{PHASE 3}

Expérience affective et dynamique représentationnelle :

- Mesure de conformité aux attentes

$\downarrow$

$\downarrow$

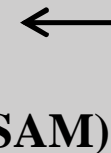

9 périodes

- Mesure discrète des affects (liste des 17 affects)

- Mesure d'attribution des affects

- Test de centralité (Test d'Indépendance au Contexte)

\section{$\sum$ Introduction $>$ Hypothèses $>$ Méthodologie}


Le plus

caractéristique

$\%$

d'Affirmation

$\mathbf{7 4 , 4 \%}$

$62,8 \%$

$65,1 \%$

$60,5 \%$

$62,8 \%$

$\mathbf{6 5 , 1 \%}$

$37,2 \%$

$39,5 \%$

$30,2 \%$

$20,9 \%$

10 dans laquelle les membres SE RECONNAISSENT les uns les autres

11 dans laquelle les membres PARTAGENT DES MÊMES IDEES ET OPINIONS

12 qui demande DES METHODES DE TRAVAIL

13 dans laquelle les membres PARTAGENT DES VALEURS COMMUNES

14 qui demande de gérer un LIEU, des HORAIRES, des OUTILS

15 qui demande d'appliquer des NORMES, des REGLES

16 dans laquelle les membres sont DEPENDANTS les uns des autres

17 qui demande de la HIERARCHIE

18 qui demande d'EXERCER DU POUVOIR
$25,6 \%$

$11,6 \%$

$11,6 \%$

$7,0 \%$

$9,3 \%$

$9,3 \%$

$7,0 \%$

$0,0 \%$
$88,4 \% *$

$88,4 \%$ *

$81,4 \%$ *

90,7\%*

$83,7 \%$ *

83,7\%*

$72,1 \%$

$65,1 \%$

$72,1 \%$

$69,8 \%$

$9,3 \%$

$76,7 \%$

$25,6 \%$

$51,2 \%$

$55,8 \%$

$11,6 \%$

$16,3 \%$

$2,3 \%$
Croyances

Périph.

\section{$\sum$ Introduction $\rangle$ Hypothèses $\rangle$ Méthodologie $\rangle$ Résultats}


- Organisation de la RS du Travail en équipe (RALM).

\section{Travailler «efficacement»,} en se «répartissant les rôles et les responsabilités», en prenant des «décisions collectives» relatives à des «objectifs communs», en «s'entraidant», et en «partageant les informations».

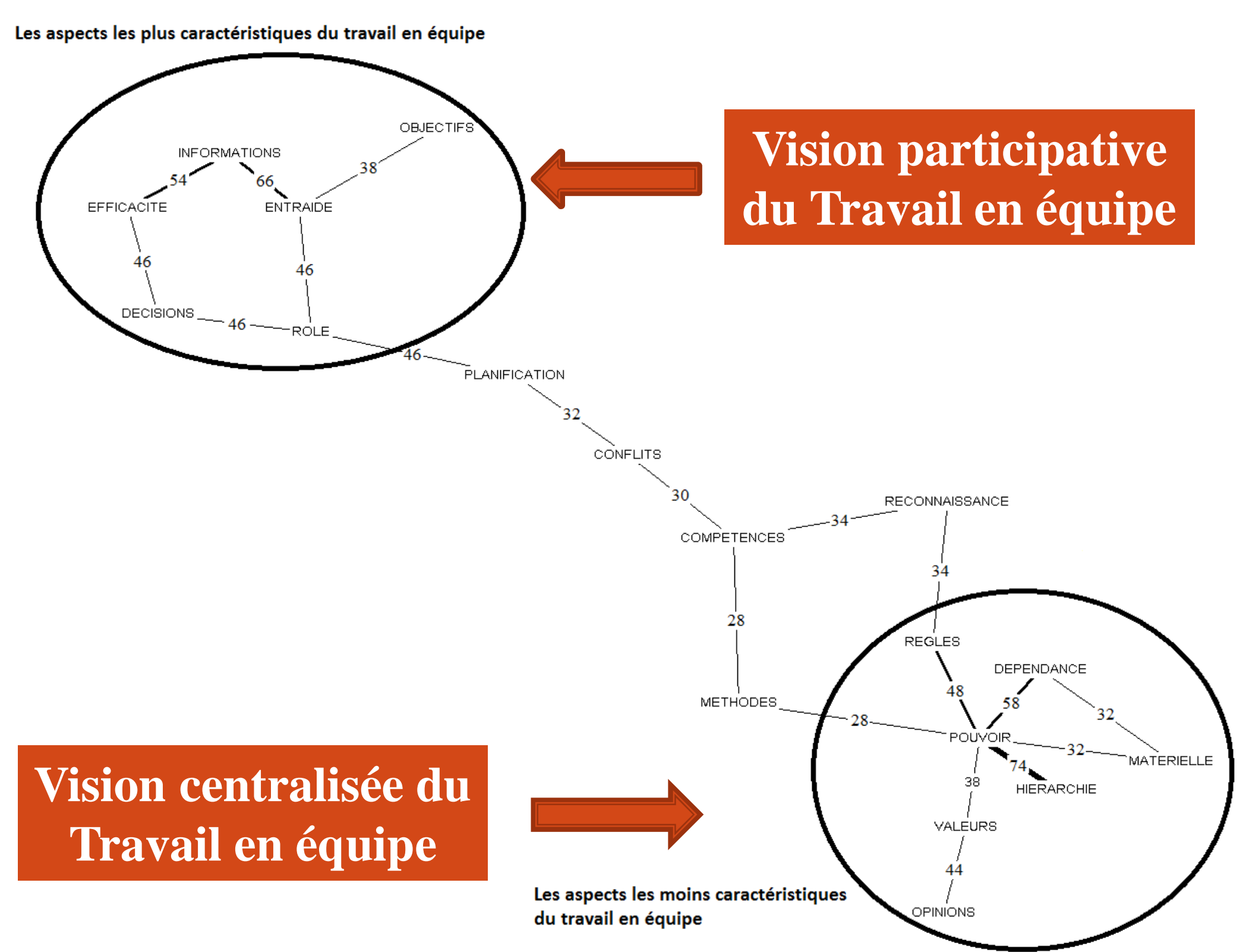

$\sum$ Introduction $\rangle$ Hypothèses $\rangle$ Méthodologie $\rangle$ Résultats 
- Expérience affective reportée (mesure discrète).

$74,7 \%$ d'affects +

\begin{tabular}{|l|c|c|}
\cline { 2 - 3 } \multicolumn{1}{c|}{} & $\mathrm{N}$ & $\%$ \\
\hline SATISFACTIS & 112 & 18,4 \\
\hline ESPOIR & 86 & 14,1 \\
\hline ENTHOUSIASME & 77 & 12,7 \\
\hline INTERET & 74 & 12,2 \\
\hline REGRET & 63 & 10,4 \\
\hline SURPRISE & 42 & 6,9 \\
\hline SOULAGEMENT & 35 & 5,8 \\
\hline JOIE & 28 & 4,6 \\
\hline SERENITE & 23 & 3,8 \\
\hline FIERTE & 19 & 3,1 \\
\hline COLERE & 17 & 2,8 \\
\hline PEUR & 11 & 1,8 \\
\hline DEGOUT & 9 & 1,5 \\
\hline CULPABILITE & 4 & 0,7 \\
\hline TRISTESSE & 4 & 0,7 \\
\hline MEPRIS & 2 & 0,3 \\
\hline HONTE & 2 & 0,3 \\
\hline
\end{tabular}

$\sum$ Introduction $\rangle$ Hypothèses $\rangle$ Méthodologie $\rangle$ Résultats


Niveau de conformité aux attentes et expérience affective reportée (mesure dimensionnelle).

H1 confirmée.

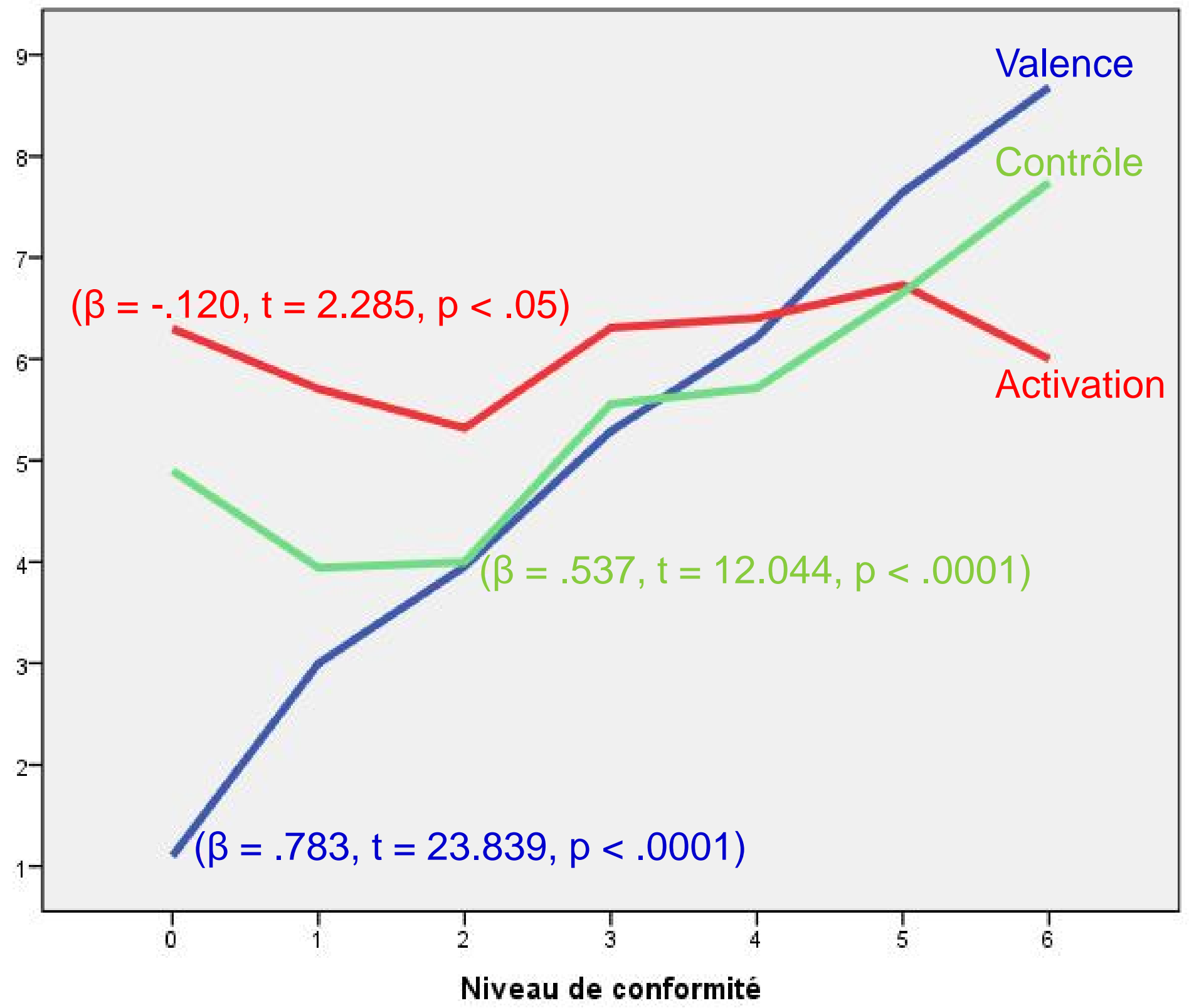

$\sum$ Introduction $\rangle$ Hypothèses $\rangle$ Méthodologie $\rangle$ Résultats


Niveau de conformité aux attentes et expérience affective reportée (mesure discrète).

H1 confirmée.

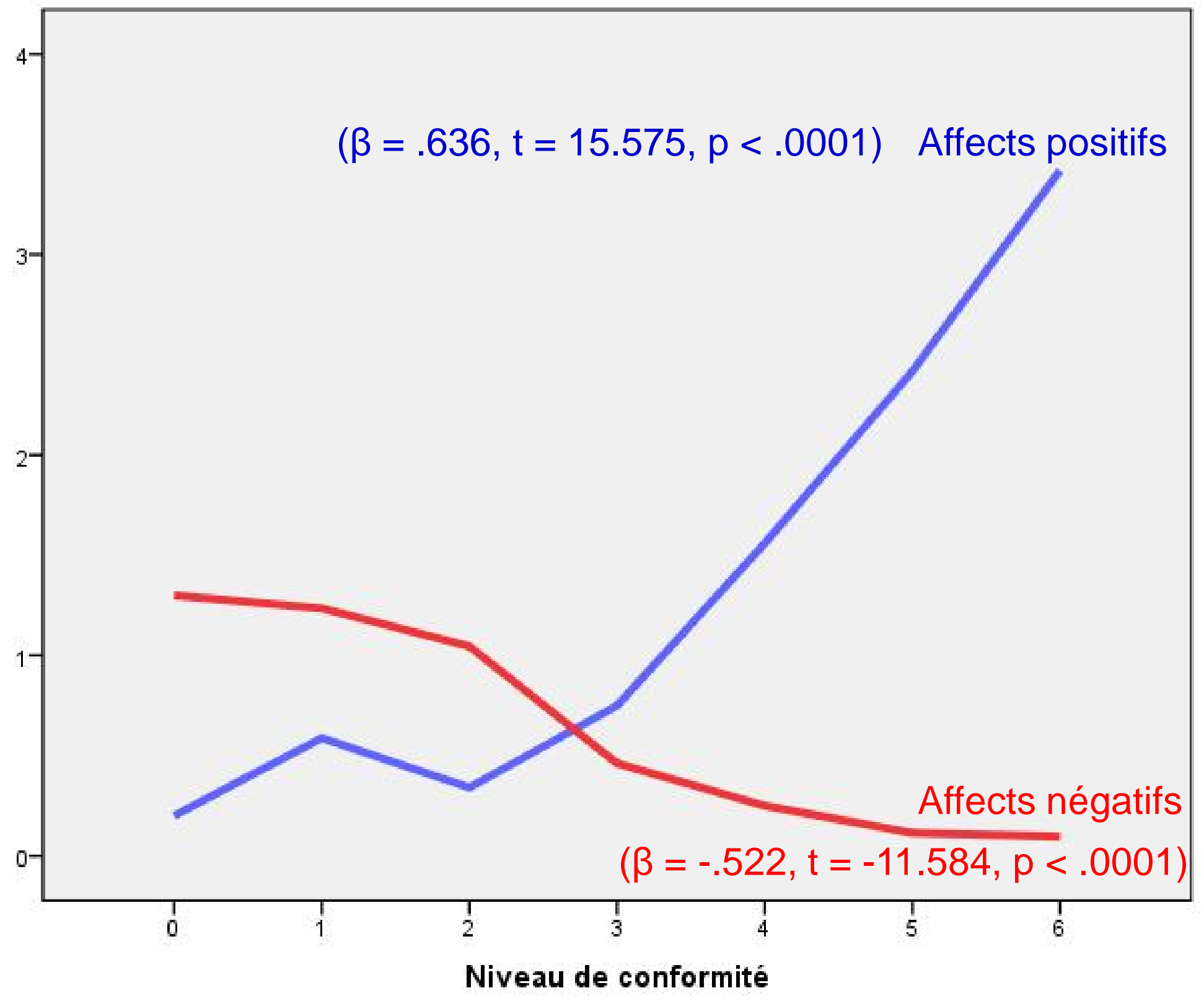


- Niveau d'activation des croyances.

H2 confirmée $(\mathrm{t}(359)=27,26, \mathrm{p}<.0001)$.

H3 confirmée.

H4 tendanciellement confirmée.

$$
\text { Croyances Centrales }
$$$$
(\beta=.240, t=4.587, p<.0001)
$$

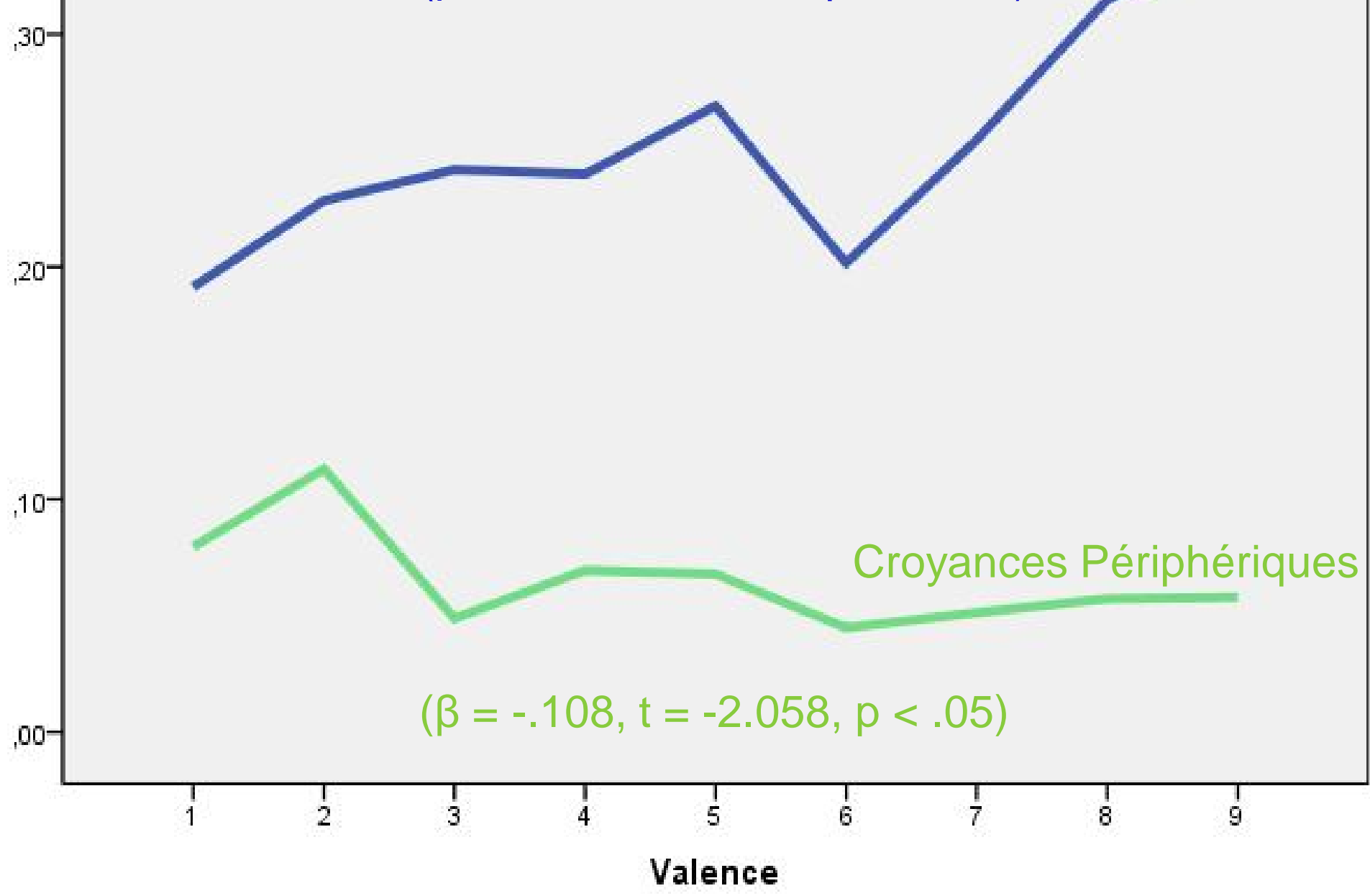

$\sum$ Introduction $\rangle$ Hypothèses $\rangle$ Méthodologie $\rangle$ Résultats 
- Opérationnalisation de la V.I.2 :

Niveau de la Valence.

\begin{tabular}{|l|c|c|}
\cline { 2 - 3 } \multicolumn{1}{l|}{ Groupes } & $\begin{array}{c}\text { Modalités de la } \\
\text { Valence }\end{array}$ & Effectif \\
\hline G0 : pré-situationnelle & Sans & 43 \\
\hline G1 : Valence Négative forte & $\ll 1 », \ll 2 »$ & 30 \\
\hline G2 : Valence Négative Modérée & $\ll 3 », \ll 4 »$ & 35 \\
\hline G3 : Valence Positive Modérée & $« 6 », \ll 7 »$ & 125 \\
\hline G4 : Valence Négative Forte & $« 8 », \ll 9 »$ & 105 \\
\hline
\end{tabular}

$\sum$ Introduction $\sum$ Hypothèses $\sum$ Méthodologie $\sum$ Résultats


Type et caractéristiques des effets.

\begin{tabular}{|c|c|}
\hline Type d'effet & Type de variations attendues \\
\hline Effet de Pratique (EP) & $\mathbf{G 0} \neq\left\{\begin{array}{l}\mathbf{G} 1 \\
\mathbf{G} 2 \\
\mathbf{G} 3 \\
\mathbf{G} 4\end{array}\right.$ \\
\hline Effet de Valence Négative (EVN) & $(\mathrm{G} 1+\mathrm{G} 2) \neq\left\{\begin{array}{l}\mathrm{G0} \\
(\mathrm{G} 3+\mathrm{G} 4)\end{array}\right.$ et $(\mathrm{G} 3+\mathrm{G} 4)=\mathrm{G0}$ \\
\hline Effet de Valence Positive (EVP) & $(\mathrm{G} 3+\mathrm{G} 4) \neq\left\{\begin{array}{l}\mathrm{G0} \\
(\mathrm{G} 1+\mathrm{G} 2)\end{array}\right.$ et $(\mathrm{G} 1+\mathrm{G} 2)=\mathrm{G0}$ \\
\hline Effet d'Intensité de Valence Négative (EIVN) & et $\mathbf{G 0}=\mathbf{G} 2=\mathbf{G 3}=\mathbf{G 4}$ \\
\hline Effet d'Intensité de Valence Positive (EIVP) & et $\quad \mathbf{G 0}=\mathbf{G 1}=\mathbf{G 2}=\mathbf{G 3}$ \\
\hline
\end{tabular}




\begin{tabular}{|c|c|c|c|c|c|c|c|}
\hline $\begin{array}{l}\text { Dynamique } \\
\text { représentationnelle }\end{array}$ & & $\begin{array}{l}\text { Initiale (a) } \\
\text { (G0) } N=43\end{array}$ & $\begin{array}{l}\text { Valence Nég. } \\
\text { forte (b) } \\
\text { (G1) } \mathbf{N}=\mathbf{3 0}\end{array}$ & $\begin{array}{l}\text { Valence Nég. } \\
\text { modérée (c) } \\
(\text { G2) } \mathbf{N}=35\end{array}$ & $\begin{array}{l}\text { Valence Pos. } \\
\text { modérée (d) } \\
(\text { G3) } \mathbf{N}=125\end{array}$ & $\begin{array}{c}\text { Valence Pos. } \\
\text { forte (e) } \\
\text { (G4) } \mathrm{N}=\mathbf{1 0 5}\end{array}$ & Effet \\
\hline \multirow{6}{*}{$\begin{array}{l}\text { H5 globalement } \\
\text { confirmée }\end{array}$} & Décisions & 88.37 & 76.67 & 88.57 & 90.40 & 92.38 & NS \\
\hline & Efficacité & 88.37 & 86.67 & 8286 & 02.00 & 90.19 & NS \\
\hline & Emtiaiute & 81.40 & 80.00 & 91.43 & 88.80 & 90.48 & NS \\
\hline & \begin{tabular}{|l|} 
Informations \\
\end{tabular} & 90.70 & 90.00 & 88.57 & 88.80 & 91.43 & $\mathbf{N S}$ \\
\hline & Rôle & 83.72 & $60.00_{\mathrm{a} * *}$ & 65.71 & $67.20_{\mathrm{a} *}$ & $63.81_{\mathrm{a} * *}$ & $\mathbf{E P}$ \\
\hline & Objectifs & 83.72 & 86.67 & $\mathbf{8 8 . 5 7}$ & $\mathbf{8 8 . 8 0}$ & 91.43 & $\mathbf{N S}$ \\
\hline \multirow[t]{4}{*}{ H6 non confirmée } & Planification & 72.09 & 63.33 & 74.29 & 72.00 & 76.19 & NS \\
\hline & \begin{tabular}{|l|} 
Conflits \\
\end{tabular} & 65.12 & $70.00_{d * e^{*}}$ & 57.14 & $52.80_{\mathrm{a}} * \mathrm{~b} *$ & $51.43_{2 * 1 *}$ & EVP \\
\hline & Compétences & 72.09 & 70.00 & 80.00 & 80.80 & 83.81 & INS \\
\hline & Reconnaissance & 69.77 & 76.67 & 62.86 & $/ 0.40$ & 69.52 & NS \\
\hline \multirow{4}{*}{$\begin{array}{l}\text { H7 partiellement } \\
\text { confirmée }\end{array}$} & Opinions & 9.30 & 43.33 $3_{\mathrm{a} * *}$ & $51.43_{\mathrm{a} * *}$ & $56.00_{\mathrm{a} * *}$ & $55.24_{a * *}$ & $\mathbf{E P}$ \\
\hline & Móthodes & 7674 & $\overline{\mathbf{0 5 . 3 3}} \mathrm{e}_{\mathrm{e}}$ & 65.71 & 68.00 & $80.00_{\mathrm{h}^{*}}$ & NS \\
\hline & \begin{tabular}{|l|} 
Valeurs \\
\end{tabular} & -45.58 & $20.00_{d^{* * *} e^{* *}}$ & $22.00_{d * *} \mathrm{e}^{*}$ & 47.20 \%** h** n** & 57.14 & EVP \\
\hline & Mos'́nielle & 51.16 & 7222 & $45 . / 1_{b^{*}}$ & $48.80_{\mathrm{h}^{* *}}$ & $47.02 \mathrm{~h}^{* *}$ & EI V IN \\
\hline \multirow[t]{4}{*}{ Effet de Valence Pos. } & Kegles & 55.81 & 66.67 $d^{* * e^{* * *}}$ & $\begin{array}{r}45.71 \\
\end{array}$ & $41.60_{\mathrm{a} * \mathrm{~h} * *}$ & $41.90_{h^{* *}}$ & $\mathbf{N S}$ \\
\hline & Dépendance & 11.63 & $60.00_{a * * e^{*}}$ & 62.86 $\mathrm{a}^{* * *} \mathrm{e}^{* *}$ & $52.80_{a^{* * *}}$ & $42.86_{a * * b * c * *}$ & $\mathbf{E P}$ \\
\hline & Hiérarchie & 16.28 & $30.00_{d * e^{* *}}$ & 14.29 & $12.80_{\mathrm{h}^{*}}$ & $0.52_{\mathbf{h} * *}$ & NS \\
\hline & Pouvoir & 2.33 & 26.67 ${ }_{\mathrm{a} * *} \mathrm{c}^{*} \mathrm{~d}^{* * *} \mathrm{e}^{* * *}$ & $8.57_{b^{*}}$ & $4.80_{\mathrm{b}^{* * *}}$ & $5.71_{\mathbf{b}^{* *}}$ & EIVN \\
\hline
\end{tabular}

\section{$\sum$ Introduction $\rangle$ Hypothèses $\rangle$ Méthodologie $\rangle$ Résultats}




\section{REPRESENTATIONS SOCIALES ET EMOTIONS}

Approche structurale : bases d'une articulation théorique entre états affectifs et RS.

Signification symbolique, affectivité et centralité.

- Rôle fondamental du système périphérique : Une RS doit sa stabilité à la capacité de son système périphérique d'endiguer les menaces symboliques potentielles envers son système central.

- Prise en compte du système périphérique : moyen de mise à jour de l'état d'activation du « processus cognitif-symbolique » (Rimé, 2005) de recherche et de production de sens résultant de l'expérience émotionnelle.

$\sum$ Introduction $\rangle$ Hypothèses $\rangle$ Méthodologie $\rangle$ Résultats $\sum$ Conclusion 